\title{
TWO REMARKS ON BLACKWELL'S THEOREM
}

\author{
EHUD LEHRER, ${ }^{* *}$ Tel Aviv University and INSEAD \\ ERAN SHMAYA, ${ }^{* * *}$ Tel Aviv University
}

\begin{abstract}
In a decision problem with uncertainty a decision maker receives partial information about the actual state via an information structure. After receiving a signal, he is allowed to withdraw and gets zero profit. We say that one structure is better than another when a withdrawal option exists if it may never happen that one structure guarantees a positive profit while the other structure guarantees only zero profit. This order between information structures is characterized in terms that are different from those used by Blackwell's comparison of experiments. We also treat the case of a malevolent nature that chooses a state in an adverse manner. It turns out that Blackwell's classical characterization also holds in this case.
\end{abstract}

Keywords: Blackwell's comparison of experiments; withdrawal option; Bayesian decision problem; minimax

2000 Mathematics Subject Classification: Primary 46N10; 62C10; 62C20; 91B06; $91 \mathrm{~B} 08$

\section{Introduction}

A decision problem is defined by a state space, a prior distribution, an action set, and a utility function. Before taking an action, a decision maker (DM) obtains partial information about the true state of nature through an information structure which randomly chooses a signal according to a probability distribution that depends on the realized state. Comparing different information structures (in the statistics literature information structures are commonly referred to as statistical experiments) has been the subject of many papers (for a comprehensive survey of this literature, see [3] or, alternatively, for a shorter review, see [2]).

We could compare the expected utility information structures yielded in a given decision problem. This comparison, however, is too specific, as one information structure may be better than another in a certain problem and worse in another. Blackwell [1] proposed a comparison that takes into account the expected utility in all possible decision problems. According to Blackwell, a structure is better than another if, whatever the decision problem, the expected utility it guarantees is higher than that guaranteed by the other structure. He showed that this partial order can be equivalently defined using three different means: stochastic matrices, expectation of convex functions, and mean-preserving stochastic maps.

Although Blackwell's partial order is quite intuitive, it is too restrictive. It requires solid data about all possible decision problems. We propose another partial order over information structures which requires less specific data and is defined on a wider range than Blackwell's order.

Received 23 January 2006; revision received 24 February 2008.

* Postal address: School of Mathematical Sciences, Tel Aviv University, Ramat Aviv, Tel Aviv, 69978, Israel.

** Email address: lehrer@post.tau.ac.il

*** Email address: gawain@post.tau.ac.il 
This note deals with a situation where a DM is given the option to withdraw upon receiving a signal without any cost (i.e. getting zero payoff). This option guarantees that the DM always gets a nonnegative payoff. A structure is said to be better than another when a withdrawal option exists if, for every decision problem, it yields a positive payoff whenever the other structure does. In other words, when a structure is better than another when a withdrawal option exists, a DM that chooses to use it will never regret doing so, as it will never result in a nonpositive profit while the other structure results in a strictly positive profit.

There is ample psychological evidence for the existence of a discrepancy between the ways in which human beings treat losses and gains. Tversky and Kahneman [4] called this phenomenon the reflection effect. From this perspective we introduce the term 'better than', which reflects a DM who primarily cares about the possibility of having regret for choosing an information structure that resulted in a loss while another could entail a positive profit. Interestingly, our ranking coincides with that of Blackwell when a withdrawal option does not exist (see Remark 1, below). However, when a withdrawal option does exist, the ranking we introduce departs from the expected utility maximization models.

In Section 3 we present a characterization of the partial order 'being better when a withdrawal option exists' by means analogous to that of Blackwell's characterization. It turns out that one structure is better than another when a withdrawal option exists, if the latter results from the former by a multiplication with a nonnegative matrix. In other words, the characterization is similar to that of Blackwell with nonnegative matrices replacing stochastic matrices. In the same vein, in our characterization, nonnegative convex functions replace convex functions, and the equality of two measures is replaced here by another relation between measures and absolute continuity.

In Section 4 we refer to a malevolent nature that chooses a state in an adverse manner. Under these circumstances, we can define an order between information structures as follows: one structure is better than another when nature is malevolent if, for every decision problem, when nature chooses a state in order to minimize payoffs, the expected utility it guarantees is higher than that guaranteed by the other. It is shown that this order coincides with 'being better than' defined by Blackwell.

\section{Decision problems with incomplete information}

Let $K$ be a finite state set. The elements of $K$ are called the states of nature. An information structure provides an agent with partial information about the actual state. When the state of nature is $k$, the agent receives a random signal $s$ whose distribution depends on $k$. Formally, an information structure is a pair $(S, \sigma)$, where $S$ is a finite set of signals and $\sigma=\left\{\sigma_{k, s}\right\}_{k \in K, s \in S}$ is a stochastic matrix. (A matrix $\left(\sigma_{k, s}\right)$ is stochastic or substochastic if $\sigma_{k, s} \geq 0$ for every $k$ and $s$ and $\sum_{s} \sigma_{k, s}=1$ or, respectively, $\sum_{s} \sigma_{k, s} \leq 1$ for every $k$.) When the actual state is $k$, the agent receives the signal $s$ with probability $\sigma_{k, s}$.

Upon getting a signal $s$, the agent needs to choose an action from a finite set $A$. If $a$ is the action taken and $k$ is the actual state, the agent receives the payoff $u(k, a)$. The payoff matrix corresponding to $A, \boldsymbol{u}$, is the matrix $(u(k, a))_{k, a}$ that has $|K|$ rows and $|A|$ columns.

A decision problem is given by $(p, A, \boldsymbol{u})$, where $p \in \Delta(K)$ is a probability distribution over $K, A$ is a finite set of actions, and $\boldsymbol{u}: K \times A \rightarrow \mathbb{R}$ is the utility function. Given an information structure $\delta=(S, \sigma)$, the decision problem is described as follows: a state of nature $k \in K$ is randomly chosen according to $p$, then the agent receives a stochastic signal according to $\&$. Given the signal, the agent chooses an action $a \in A$ and receives payoff $u(k, a)$. Denote by $R(\delta ; p, A, \boldsymbol{u})$ the best payoff the agent can receive in the decision problem. 
Definition 1. We say that $\delta$ is better than $\mathcal{T}$ if, for every decision problem $(p, A, \boldsymbol{u})$, $R(\mathcal{T} ; p, A, \boldsymbol{u})>0$ implies that $R(\delta ; p, A, \boldsymbol{u})>0$.

Remark 1. (i) If $R(\& ; p, A, \boldsymbol{u}) \geq R(\mathcal{T} ; p, A, \boldsymbol{u})$ for every $p, A$, and $\boldsymbol{u}$ then $\delta$ is better than $\mathcal{T}$. Conversely, assume that $\delta$ is better than $\mathcal{T}$. If $R(\mathcal{S} ; p, A, \boldsymbol{u})<R(\mathcal{T} ; p, A, \boldsymbol{u})$ for some $p, A$, and $\boldsymbol{u}$ then define a new utility function $\boldsymbol{u}^{\prime}=\boldsymbol{u}-R(\mathcal{s} ; p, A, \boldsymbol{u})$. We obtain $R\left(\delta ; p, A, \boldsymbol{u}^{\prime}\right)=0<R(\mathcal{T} ; p, A, \boldsymbol{u})$, which is a contradiction. Thus, saying that $\delta$ is better than $\mathcal{T}$ is equivalent to saying that $R(\delta ; p, A, \boldsymbol{u}) \geq R(\mathcal{T} ; p, A, \boldsymbol{u})$ for every $p, A$, and $\boldsymbol{u}$.

(ii) Let $p_{0}$ be the uniform distribution over $K$. Note that if $R\left(\mathcal{s} ; p_{0}, A, \boldsymbol{u}\right) \geq R\left(\mathcal{T} ; p_{0}, A, \boldsymbol{u}\right)$ for every $A$ and $\boldsymbol{u}$ then $R(\delta ; p, A, \boldsymbol{u}) \geq R(\mathcal{T} ; p, A, \boldsymbol{u})$ for every $p, A$, and $\boldsymbol{u}$. Indeed, fix $p$, $A$, and $\boldsymbol{u}$ and define $u_{0}(k, a)=u(k, a) p(k) / p_{0}(k)$. Then,

$$
R(\& ; p, A, \boldsymbol{u})=R\left(\& ; p_{0}, A, \boldsymbol{u}_{0}\right) \geq R\left(\mathcal{T} ; p_{0}, A, \boldsymbol{u}_{0}\right)=R(\mathcal{T} ; p, A, \boldsymbol{u}) .
$$

Every information structure $\delta=(S, \sigma)$ induces a probability measure $m_{\sigma}$ over $\Delta(K)$ in the following way. Consider the probability space $K \times S$ equipped with the probability measure $p(k, s)=(1 /|K|) \sigma_{k, s}$. Then the posterior distribution of $k$ given $s$ is a $\Delta(K)$-valued random variable defined over this probability space. We denote by $m_{\sigma}$ the distribution of this random variable and call it the standard measure associated with $\$$. This is a probability measure with finite support. Its atoms are the normalized columns of $\sigma$.

A stochastic transformation over $\Delta(K)$ is a function $T(x, E)$ defined for every $x \in \Delta(K)$ and a Borel subset $E$ of $\Delta(K)$ such that $E \mapsto T(x, E)$ is a probability measure over $\Delta(K)$ for every $x \in \Delta(K)$, and such that $x \mapsto T(x, E)$ is measurable for every Borel subset $E$ of $\Delta(K)$. For every probability measure $m$ over $\Delta(K)$, the function $M(E)=\int T(x, E) \mathrm{d} m(x)$ is a probability measure over $\Delta(K)$. We denote $M=T m$. We call $T$ mean preserving if $\int y T(x, \mathrm{~d} y)=x$ for every $x \in \Delta(K)$. The following theorem is due to Blackwell [1].

Theorem 1. ([1].) Let $\&=(S, \sigma)$ and $\mathcal{T}=(T, \tau)$ be two information structures. Then the following conditions are equivalent.

(a) For every $p$, $A$, and $\boldsymbol{u}$, if $R(\mathcal{T} ; p, A, \boldsymbol{u})>0$ then $R(\& ; p, A, \boldsymbol{u})>0$.

(b) There exists a stochastic matrix $\varepsilon=\left\{\varepsilon_{s, t}\right\}_{s \in S, t \in T}$ such that $\boldsymbol{\tau}=\sigma \boldsymbol{\varepsilon}$, the product of the matrices $\boldsymbol{\sigma}$ and $\boldsymbol{\varepsilon}$.

(c) There exists a mean-preserving stochastic map $T$ over $\Delta(K)$ such that $T m_{\tau}=m_{\sigma}$.

(d) For every convex and continuous function $h: \Delta(K) \rightarrow \mathbb{R}$, if $\int h \mathrm{~d} m_{\tau}>0$ then $\int h \mathrm{~d} m_{\sigma}>0$.

\section{Decision problems with a withdrawal option}

Assume that, after having received a signal, the agent is allowed to withdraw and to obtain zero payoff. Formally, the decision problem $(p, A, \boldsymbol{u})$ with a withdrawal option is the decision problem $\left(p, A^{0}, \boldsymbol{u}\right)$, where $A^{0}=A \cup\{\underline{0}\}$ and $u(k, \underline{0})=0$ for every $k \in K$. Denote by

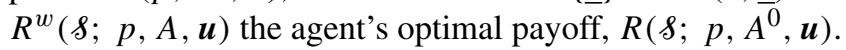

A DM who needs to choose between obtaining information via $\delta$ or via $\mathcal{T}$ before knowing the payoff function has no problem when $\delta$ is better than $\mathcal{T}$. However, the 'better than' order is not complete and quite often neither $\delta$ is better than $\mathcal{T}$ nor $\mathcal{T}$ is better than $\delta$. However, suppose that a withdrawal option is available. Moreover, suppose that the only information about the information structures is that whenever a positive profit is guaranteed when getting 
signals through $\mathcal{T}$, it is always the case when getting signals through $\delta$. In the sense that it may never happen that getting signals through $\mathcal{T}$ ensures a positive profit while getting signals through $\&$ ensures only zero profit, i.e. $\&$ is better than $\mathcal{T}$. This order is formally defined as follows.

Definition 2. We say that $\delta$ is better than $\mathcal{T}$ when a withdrawal option exists if, for every decision problem $(p, A, \boldsymbol{u}), R^{w}(\mathcal{T} ; p, A, \boldsymbol{u})>0$ implies that $R^{w}(s ; p, A, \boldsymbol{u})>0$.

It is clear that, when $\&$ is better than $\mathcal{T}$ when a withdrawal option exists, $\&$ is better than $\mathcal{T}$. Theorem 1 states that $\delta$ is better than $\mathcal{T}$ if and only if there exists a stochastic matrix $\boldsymbol{\varepsilon}$ such that $\tau=\sigma \varepsilon$. In the following theorem the fact that $\delta$ is better than $\mathcal{T}$ when a withdrawal option exists is characterized by weaker conditions than that of Theorem 1. For instance, $\boldsymbol{\tau}=\boldsymbol{\sigma} \boldsymbol{\varepsilon}$, where $\varepsilon$ is merely a matrix whose entries are nonnegative.

Example 1. Let the number of states of nature be four, and let $\sigma$ and $\boldsymbol{\tau}$ be given by

$$
\boldsymbol{\sigma}=\left(\begin{array}{cccc}
\frac{1}{2} & 0 & \frac{1}{2} & 0 \\
\frac{1}{2} & 0 & 0 & \frac{1}{2} \\
0 & \frac{1}{2} & \frac{1}{2} & 0 \\
0 & \frac{1}{2} & 0 & \frac{1}{2}
\end{array}\right) \quad \text { and } \quad \boldsymbol{\tau}=\left(\begin{array}{cc}
1 & 0 \\
1 & 0 \\
0 & 1 \\
0 & 1
\end{array}\right)
$$

There exists no stochastic matrix $\varepsilon$ such that $\tau=\sigma \varepsilon$. However, denoting,

$$
\boldsymbol{\varepsilon}=\left(\begin{array}{ll}
2 & 0 \\
0 & 2 \\
0 & 0 \\
0 & 0
\end{array}\right),
$$

we obtain $\boldsymbol{\tau}=\boldsymbol{\sigma} \boldsymbol{\varepsilon}$. Thus, there exists a matrix $\boldsymbol{\varepsilon}$ with nonnegative entries such that $\boldsymbol{\tau}=\boldsymbol{\sigma} \boldsymbol{\varepsilon}$ and, by Theorem 2, below, the information structure corresponding to $\sigma$ is better than that corresponding to $\boldsymbol{\tau}$ when a withdrawal option exists.

Theorem 1(a) means that there exists a stochastic transformation (i.e. a linear transformation that maps probability measures over $S$ to probability measures over $T$ ) which maps the $k$ th row of $\sigma$ to the $k$ th row of $\boldsymbol{\tau}$. Theorem 2, below, is analogous to Theorem 1. It characterizes when one information structure is better than another when a withdrawal option exists. However, instead of using terms of stochastic matrices, it uses terms of matrices that have nonnegative entries.

A matrix $\varepsilon=\left\{\varepsilon_{s, t}\right\}_{s \in S, t \in T}$ with nonnegative entries induces a linear transformation that maps measures (not necessarily probability) over $S$ to measures over $T$. The fact that $\tau=\sigma \varepsilon$, with $\varepsilon$ being a matrix with nonnegative entries, means that there exists such a transformation that maps the $k$ th row of $\sigma$ (which is a probability measure of $S$ ) to the $k$ th row of $\tau$ (a probability measure over $T$ ).

Remark 2. The appearance of the stochastic matrix $\boldsymbol{\varepsilon}$ in Theorem 1(b) has a natural probabilistic interpretation: the agent receives a signal $s \in S$ and then simulates a new signal $t \in T$ (the distribution of $t$ is dictated by $s$ ). The more general notion of maps between measures cannot be interpreted in such a way. In some sense, the equivalence between conditions (a) and (b) of Theorem 2, below, establishes the operational meaning of these maps, since it characterizes the cases when one information structure can be transformed to another in terms of the best payoff each structure yields in games. 
Theorem 2. The following conditions are equivalent.

(a) $\&$ is better than $\mathcal{T}$ when a withdrawal option exists.

(b) There exists a matrix $\boldsymbol{\varepsilon}$ with nonnegative entries such that $\boldsymbol{\tau}=\boldsymbol{\sigma} \boldsymbol{\varepsilon}$.

(c) For every convex, continuous, and nonnegative function $h: \Delta(K) \rightarrow \mathbb{R}$, if $\int h \mathrm{~d} m_{\tau}>0$ then $\int h \mathrm{~d} m_{\sigma}>0$.

(d) There exists a mean-preserving stochastic map $T$ over $\Delta(K)$ such that $T m_{\tau}$ is absolutely continuous with respect to $m_{\sigma}$.

Proof. Firstly, we prove that condition (a) implies condition (b). Assume that there exists no matrix $\boldsymbol{\varepsilon}$ with nonnegative entries such that $\boldsymbol{\tau}=\boldsymbol{\sigma} \boldsymbol{\varepsilon}$. In particular, there exists some column $\boldsymbol{\tau}_{* t}$ of $\boldsymbol{\tau}$ that is not a conic combination of the columns of $\sigma$ (a conic combination of vectors is a linear combination with nonnegative coefficients). By the separation theorem, there exists a $|K| \times 1$ matrix $\boldsymbol{\alpha}$ such that $\left\langle\boldsymbol{\tau}_{* t}, \boldsymbol{\alpha}\right\rangle>0$ but $\langle\boldsymbol{\sigma} \boldsymbol{\varepsilon}, \boldsymbol{\alpha}\rangle \leq 0$ for every $|S| \times 1$ matrix $\boldsymbol{\varepsilon}$ with nonnegative coefficients (for matrices $\boldsymbol{X}$ and $\boldsymbol{Y}$ of the same dimension, we define $\langle\boldsymbol{X}, \boldsymbol{Y}\rangle=\operatorname{tr}\left(\boldsymbol{X}^{\top} \boldsymbol{Y}\right)=$ the sum of the entries along the main diagonal of the matrix $\boldsymbol{X}^{\top} \boldsymbol{Y}$ ). (Note that the action set $A$ corresponding to $\alpha$ is a singleton.) Let $p$ be the uniform distribution over $K$.

Consider the strategy (in the game played under $\boldsymbol{\tau}$ ) that prescribes playing action $a$ when obtaining signal $t$, and withdrawing otherwise. The expected payoff of this strategy is $\left(\sum_{k} \boldsymbol{\tau}_{k t} /|K|\right)\left\langle\boldsymbol{\tau}_{* t}, \boldsymbol{\alpha}\right\rangle>0$. Thus, $R^{w}(\mathcal{T} ; p, A, \boldsymbol{u})>0$. However, the expected payoff of any strategy $\boldsymbol{\gamma}$ is $\langle\boldsymbol{\sigma} \boldsymbol{\gamma}, \boldsymbol{\alpha}\rangle \leq 0$. This means that $R^{w}(8 ; p, A, \boldsymbol{u})=0$, which proves the desired assertion.

Secondly, we prove that condition (c) implies condition (b). If there exists no matrix $\boldsymbol{\varepsilon}$ with nonnegative entries such that $\boldsymbol{\tau}=\sigma \varepsilon$, let $\boldsymbol{\alpha}$ be as in the previous paragraph and define $h(\boldsymbol{x})=\max (\boldsymbol{x} \boldsymbol{\alpha}, 0)(\boldsymbol{\alpha}$ is a $|K|$-dimensional vector and $\boldsymbol{x} \boldsymbol{\alpha}$ the inner product of $\boldsymbol{x}$ and $\boldsymbol{\alpha})$. The function $h$ is nonnegative and as a maximum of two linear functions, it is convex. Finally, the properties of $\boldsymbol{\alpha}$ imply that $\int h \mathrm{~d} m_{\tau}>0$ but $\int h \mathrm{~d} m_{\sigma}=0$, contrary to condition (c).

Thirdly, we prove that condition (b) implies condition (a). Let $A$ be the set of actions. A strategy of the agent is given by a substochastic matrix $\gamma=\left\{\gamma_{s, a}\right\}_{s \in S, a \in A}$ : if the signal is $s$, the player takes action $a$ with probability $\gamma_{s, a}$. Assume, without loss of generality, that $p$ is the uniform distribution over $K$. Then the expected payoff to the player is given by $(1 /|K|)\langle\boldsymbol{\tau} \boldsymbol{\gamma}, \boldsymbol{\alpha}\rangle$, where $\boldsymbol{\alpha}$ is the payoff matrix corresponding to $A$ and $\boldsymbol{u}$. Assume that this is strictly greater than 0 . By assumption, $\boldsymbol{\tau}=\boldsymbol{\sigma} \boldsymbol{\varepsilon}$ for some matrix $\boldsymbol{\varepsilon}$ with nonnegative entries. Thus, $\boldsymbol{\varepsilon} \boldsymbol{\gamma}=C \boldsymbol{\gamma}^{\prime}$ for some substochastic matrix $\boldsymbol{\gamma}^{\prime}$ and a constant $C>0$. If the agent uses the strategy $\boldsymbol{\gamma}^{\prime}$ in the $\&$-game then his payoff is

$$
R^{w}(8 ; p, A, \boldsymbol{u})=\frac{1}{|K|}\left\langle\boldsymbol{\sigma} \boldsymbol{\gamma}^{\prime}, \boldsymbol{\alpha}\right\rangle=\frac{1}{C|K|}\langle\boldsymbol{\tau} \boldsymbol{\gamma}, \boldsymbol{\alpha}\rangle=\frac{1}{C} R^{w}(\mathcal{T} ; p, A, \boldsymbol{u})>0 .
$$

Fourthly, we prove that condition (b) implies condition (d). Let $\boldsymbol{x}$ be an atom of $\mu_{\boldsymbol{\tau}}$, which corresponds to a column of $\boldsymbol{\tau}$. By condition (b), this column is a conic combination of some columns of $\boldsymbol{\sigma}$. It follows that $\boldsymbol{x}$ is in the convex hull of the atoms of $m_{\sigma}$. Therefore, there exists a probability measure, $\mu_{x}$, over $\Delta(K)$ which is absolutely continuous with respect to $m_{\sigma}$ such that $\boldsymbol{x}=\int y \mathrm{~d} \mu_{\boldsymbol{x}}$. We let $T(\boldsymbol{x}, E)=\mu_{\boldsymbol{x}}(E)$ for every atom $\boldsymbol{x}$ of $m_{\boldsymbol{\tau}}$ and $T(\boldsymbol{x}, E)=\delta_{\boldsymbol{x}}(E)$ for every $\boldsymbol{x}$ outside the support of $m_{\boldsymbol{\tau}}$. Here, $\delta_{\boldsymbol{x}}$ is Dirac's atomic measure at $\boldsymbol{x}$. Then $T$ is mean preserving and $T \boldsymbol{\tau}$ is absolutely continuous with respect to $\sigma$.

Finally, we prove that condition (d) implies condition (c). Let $h: \Delta(K) \rightarrow \mathbb{R}$ be a convex, continuous, and nonnegative function such that $\int h \mathrm{~d} m_{\tau}>0$. Since $h$ is convex and $T$ is 
mean preserving, it follows that $\int h \mathrm{~d} T m_{\tau} \geq \int h \mathrm{~d} m_{\tau}>0$. Since $h$ is nonnegative and $T m_{\tau}$ is absolutely continuous with respect to $m_{\sigma}$, it follows that $\int h \mathrm{~d} m_{\sigma}>0$.

Remark 3. Unlike the case without a withdrawal option, Theorem 2(b) does not imply that, for every decision problem $(p, A, \boldsymbol{u}), R^{w}(\mathcal{T} ; p, A, \boldsymbol{u}) \geq R^{w}(\delta ; p, A, \boldsymbol{u})$. This is equivalent to $R(\mathcal{T} ; p, A, \boldsymbol{u}) \geq R(\& ; p, A, \boldsymbol{u})$ for every decision problem $(p, A, \boldsymbol{u})$. Indeed, by adding a large positive constant $M$ to $\boldsymbol{u}$, the withdrawal option becomes irrelevant. Therefore, if, for every decision problem $(p, A, \boldsymbol{u}), R^{w}(\mathcal{T} ; p, A, \boldsymbol{u}) \geq R^{w}(s ; p, A, \boldsymbol{u})$, then $R(\mathcal{T} ; p, A, \boldsymbol{u}+M)=$ $R^{w}(\mathcal{T} ; p, A, \boldsymbol{u}+M) \geq R^{w}(\delta ; p, A, \boldsymbol{u}+M)=R(\& ; p, A, \boldsymbol{u}+M)$ for every $(p, A, \boldsymbol{u})$. This implies that $R(\mathcal{T} ; p, A, \boldsymbol{u}) \geq R(\mathcal{\delta} ; p, A, \boldsymbol{u})$ for every $(p, A, \boldsymbol{u})$.

Remark 4. It follows from Theorem 2 that $\delta$ and $\mathcal{T}$ are equivalent when a withdrawal option exists (that is, $R^{w}(\mathcal{T} ; p, A, \boldsymbol{u})>0$ is equivalent to $R^{w}(8 ; p, A, \boldsymbol{u})>0$ for every $p, A$, and $\boldsymbol{u}$ ) if and only if the supports of the corresponding standard measures $m_{\sigma}$ and $m_{\tau}$ have the same convex hull.

\section{A malevolent nature}

In Section 3 we let the DM have an extra withdrawal option. In this section we let Nature have an extra power. Consider a situation in which Nature chooses her state strategically to minimize the agent payoff. An information structure $\delta=(s, \sigma)$ and a set of actions $A$ induce a zero-sum game played by the agent (the maximizer) and Nature (the minimizer). Nature chooses a state $k$, then a signal $s$ is chosen according to $\&$, the agent is informed of it and chooses an action $a$. The payoff is $u(k, a)$. Let

$$
R_{m}(\& ; A, \boldsymbol{u})=\min _{p \in \Delta(K)} R(\delta ; p, A, \boldsymbol{u})
$$

be the value of this game. The value exists since each player has finitely many strategies. It turns out that the partial order induced over information structures when Nature is malevolent coincides with that of Theorem 1. Formally, we have the following theorem.

Theorem 3. Let $\delta=(S, \sigma)$ and $\mathcal{T}=(T, \tau)$ be two information structures. Then the following conditions are equivalent.

(a) For every $A$, if $R_{m}(\mathcal{T} ; A, \boldsymbol{u})>0$ then $R_{m}(8 ; A, \boldsymbol{u})>0$.

(b) There exists a stochastic matrix $\varepsilon=\left\{\varepsilon_{s, t}\right\}_{s \in S, t \in T}$ such that $\boldsymbol{\tau}=\boldsymbol{\sigma} \boldsymbol{\varepsilon}$.

Proof. First we prove that condition (b) implies condition (a). Suppose that $\boldsymbol{\tau}=\sigma \boldsymbol{\varepsilon}$ for a stochastic matrix $\varepsilon$, and let $A$ be a finite set of actions such that $R_{m}(\mathcal{T} ; A, \boldsymbol{u})>0$. If $p \in \Delta(K)$ is an optimal strategy for Nature in the $\&$-game then $R(\mathcal{T} ; p, A, \boldsymbol{u}) \geq R_{m}(\mathcal{T} ; A, \boldsymbol{u})>0$. By Theorem 1,

$$
R_{m}(\& ; A, \boldsymbol{u})=R(\& ; p, A, \boldsymbol{u})>0 .
$$

Now we prove that condition (a) implies condition (b). Assume that there exists no stochastic matrix $\boldsymbol{\varepsilon}=\left\{\varepsilon_{s, t}\right\}_{s \in S, t \in T}$ such that $\boldsymbol{\tau}=\boldsymbol{\sigma} \boldsymbol{\varepsilon}$. Let $Q_{1}$ be the set of all matrices of the form $\boldsymbol{\sigma} \boldsymbol{\varepsilon}$, where $\varepsilon$ ranges over all stochastic matrices $\varepsilon=\left\{\varepsilon_{s, t}\right\}_{s \in S, t \in T}$. Let $Q_{2}$ be the set of all matrices of the form $\delta \boldsymbol{\tau}$, where $\delta$ is a diagonal $|K| \times|K|$ matrix with nonnegative entries. Here $Q_{1}$ is a compact convex set and $Q_{2}$ is a convex cone in the vector space of all $|K| \times|T|$ matrices.

First assume that $Q_{1} \cap Q_{2} \neq \varnothing$. Let $\delta \boldsymbol{\tau} \in Q_{2}$ also belong to $Q_{1}$. As any matrix in $Q_{1}, \boldsymbol{\delta} \boldsymbol{\tau}$ is stochastic. This may happen only if $\delta$ is the identity matrix, in which case $\tau \in Q_{1}$, that is, $\tau=\sigma \varepsilon$ and condition (b) is satisfied. 
Now assume that $Q_{1} \cap Q_{2} \neq \varnothing$. By the separation theorem, there exists a $|K| \times|T|$ matrix $\boldsymbol{\alpha}$ that strictly separates $Q_{1}$ and $Q_{2}$. That is, $\langle\boldsymbol{\alpha}, \boldsymbol{x}\rangle<0$ for every $\boldsymbol{x} \in Q_{1}$ and $\langle\boldsymbol{\alpha} \boldsymbol{x}\rangle>0$ for every $\boldsymbol{x} \in Q_{2}$. Define $A$ to be the set of the columns of $\boldsymbol{\alpha}$, and let $\boldsymbol{u}$ be the utility function that turns $\boldsymbol{\alpha}$ into the payoff matrix that corresponds to $A$ and $\boldsymbol{u}$.

First suppose that the game is played under $\delta$, and that Nature's mixed strategy is uniform over $K$. Let an agent's strategy be given by a stochastic matrix $\varepsilon=\left\{\varepsilon_{s, t}\right\}_{s \in S, t \in T}$ (that is, when he receives the signal $s$, he chooses the $t$-column of $\boldsymbol{\alpha}$ with probability $\varepsilon_{s, t}$ ). The agent's payoff is then $(1 /|K|)(\sigma \varepsilon) \boldsymbol{\alpha}$, which is strictly smaller than 0 since $\sigma \varepsilon \in Q_{1}$. It follows that $R_{m}(8 ; A)<0$.

Now suppose that the game is played under $\mathcal{T}$, and that the agent's strategy prescribes him to play the signal he received. If Nature picks state $k$ (it is a pure strategy of Nature) then the payoff is $\left\langle\boldsymbol{\eta}_{k} \boldsymbol{\tau}, \boldsymbol{\alpha}\right\rangle$, where $\boldsymbol{\eta}_{k}$ is the $|K| \times|K|$ matrix whose only nonzero entry is the $(k, k)$ th entry, which is 1 . Since $\boldsymbol{\eta}_{k} \boldsymbol{\tau} \in Q_{2}$ for every $k$, it follows that the agent has a positive payoff against every pure strategy of Nature and, therefore, against any of its mixed strategies. Thus, $R_{m}(\mathcal{T} ; A)>0$.

\section{Final remarks}

\subsection{Different withdrawal options}

Assume that instead of a constant zero payoff, the withdrawal option yields a payoff $\boldsymbol{b} \in \mathbb{R}^{K}$ that depends on the realized state of nature. For every decision problem $(p, A, \boldsymbol{u})$ and information structure $\delta$, denote by $R^{w}(\delta ; p, A, \boldsymbol{u} ; \boldsymbol{b})$ the DM's optimal payoff if he is allowed the withdrawal option $\boldsymbol{b}$. It turns out that Theorem 2 can be stated as follows.

Theorem 4. (a) There exists a matrix $\boldsymbol{\varepsilon}$ with nonnegative entries such that $\boldsymbol{\tau}=\boldsymbol{\sigma} \boldsymbol{\varepsilon}$. This condition implies the following condition.

(b) For every decision problem $(p, A, \boldsymbol{u})$, if $R^{w}(\mathcal{T} ; p, A, \boldsymbol{u} ; \boldsymbol{b})>R(\mathcal{T} ; \boldsymbol{b})$ then $R^{w}(8 ; p$, $A, \boldsymbol{u} ; \boldsymbol{b})>R(\& ; \boldsymbol{b})$.

We do not know the analogous condition in the case of several withdrawal options.

\subsection{General measures and signal spaces}

The results above can be stated in more general terms, not necessarily with finite signal spaces. In order to keep this note concise, we choose to omit the details.

\section{References}

[1] Blackwell, D. (1953). Equivalent comparisons of experiments. Ann. Math. Statist. 24, 265-272.

[2] Le CAM, L. (1996). Comparison of experiments-a short review. In Statistics, Probability and Game Theory (IMS Lecture Notes Monogr. Ser. 30), eds T. Ferguson and L. Shapley, Institute of Mathematical Statistics, Hayward, CA, pp. 127-138.

[3] Torgersen, E. N. (1991). Comparison of Statistical Experiments. Cambridge University Press.

[4] Tversky A. And Kahneman, D. (1981). The framing of decisions and the psychology of choice. Science 211, $453-458$. 